\title{
Time Aggregation for Network Design to Meet Time-Constrained Demand
}

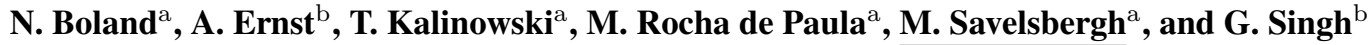

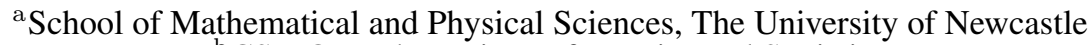 \\ ${ }^{\mathrm{b}}$ CSIRO Mathematics, Informatics and Statistics \\ Email: Martin.Savelsbergh@newcastle.edu.au
}

\begin{abstract}
:
We study a network design problem inspired by a strategic planning problem encountered in the Hunter Valley Coal Chain. Demand is given in the form of freight that is available from a specific date and has to be transported from multiple origins to a single destination before its deadline. It is possible to temporarily store freight at certain intermediate locations along the way from origins to destination. The objective is to determine minimum-cost capacity expansions required on the links and nodes of the network, if any, so as to be able to transport all freight within its given time windows.

A natural mixed integer programming formulation with a daily granularity quickly becomes computationally intractable. We investigate the potential of time aggregation to overcome the computational challenges. By aggregating consecutive time periods, a smaller instance is obtained, which can be solved more easily and provides a lower bound on the optimal value.

A carefully designed iterative disaggregation scheme identifies a time aggregation that yields an optimal solution to the original problem. An extensive computational study demonstrates the efficacy of the proposed approach.
\end{abstract}

Keywords: Network design problem, integer programming, time indexed models, time aggregation 


\section{INTRODUCTION}

In this research, we study a general class of network design problems that exhibit some of the essential features of the strategic planning problem encountered at the Hunter Valley Coal Chain (HVCC).

Consider a network with a set of nodes $N$ and a set of $\operatorname{arcs} A$ and a set of commodities $C$ that have to be transported over the network. The freight of commodity $j \in C$ can be temporarily stored in certain nodes $N_{j}^{S} \subseteq N$. Each commodity has an associated demand $F_{j}$ that has to flow from a set of sources $Q_{j} \in N_{j}^{S}$ to a single destination $s_{j} \in N_{j}^{S}$. Each of the sources $q \in Q_{j}$ has an associated contribution $f_{j}^{q}$ to the total demand. The freight for commodity $j \in C$ is available at its sources at release date $r_{j}$ and must reach its destination at or before a deadline $d_{j}$. Each arc $a \in A$ has a current capacity $u_{a}$ that can be expanded to a maximum of $u_{a}^{+}$for a fixed cost of $c_{a}^{1}$ plus $c_{a}^{2}$ per unit of installed capacity. Similarly, every storage node $v \in\left(N^{S}=\bigcup_{j \in C} N_{j}^{S}\right)$ has also a current capacity $u_{v}$ that can be expanded to a maximum of $u_{v}^{+}$for a fixed cost of $c_{v}^{1}$ plus $c_{v}^{2}$ per unit of installed capacity. The objective is to determine minimum-cost capacity expansions required on the links and nodes of the network, if any, so as to be able to transport all freight within its given time windows.

Due to their great importance to industry, Capacity Expansion Problems (CEPs) have been studied since the 60's, with many different applications, as shown in an early survey published by Luss (1982). Ordóñez and Zhao (2007) consider the expansion of arc capacities in a network to minimize the linear transportation cost in a network with uncertain data. Ahmed et al. (2003) consider a similar problem, exploiting the lot sizing substructure of their problem, also in an uncertain environment, to minimize the cost of cost of the expansions.

In section 2, we formulate the CEP using a natural Mixed-Integer Programming (MIP) model considering discrete time periods. As discussed by Floudas and Lin (2004), when using this kind of time representation, it is usually necessary to use time periods that are sufficiently small to achieve a suitable approximation of the real world problem. That, along with the use of long time horizons, leads to very large optimization problems which are often intractable.

To cope with that, Newman and Kuchta (2007) collapsed adjacent time periods into stages with the same duration (the same number of time periods) to reduce the size of a model used to schedule iron ore production over multiple discrete time periods. In this research, we generalize this idea, proposing the use of smaller problems that aggregate an arbitrary number of time periods, to obtain lower bounds for the problem.

In section 3 , we propose a carefully designed iterative disaggregation scheme to identify a time aggregation that yields an optimal solution to the original problem.

Finally, in section 4 we present computational results obtained with the proposed disaggregation schemes on instances derived from real HVCC data, and conclude with some final remarks in section 5.

\section{A NATURAL MIP FORMULATION WITH DISCRETE AGGREGATED TIME PERIODS}

In this research, the proposed models consider that the time horizon $T=\left[\min _{j \in C}\left(r_{j}\right), \max _{j \in C}\left(d_{j}\right)\right]$ is discretized in $\left(\max _{j \in C}\left(d_{j}\right)-\min _{j \in C}\left(r_{j}\right)+1\right)$ time periods with the same duration.

Define a time aggregation a set of adjacent time periods. Let a breakpoint be the first time period of a time aggregation and define $\beta \subseteq T$ as the ordered list of such breakpoints. Let $\bar{t} \in \beta=\{\ldots, t, \bar{t}, \ldots\}$ be the breakpoint found immediately after breakpoint $t$ in $\beta$. A time aggregation is thus defined by two consecutive breakpoints $[t, \bar{t}) \in \beta$.

Formulation 1 formulates the CEP accounting for time aggregations.

In this model, the binary design decision variables $y_{a}$ and $y_{v}$ are set to 1 if we have to install extra capacity on arc $a \in A$ or storage node $v \in N^{S}$, respectively, and 0 otherwise. The design decision variables $z_{a}$ and $z_{v}$ hold the extra capacity installed on arc $a$ or storage node $v$, respectively. The operational decision variables $\phi_{a j}^{t}$ and $\phi_{v j}^{t}$ hold the flow of commodity $j \in C$ at time $t \in\left[r_{j}, d_{j}\right]$ on arc $a$ or storage node $v$, respectively.

Constraints (2) and (3) ensure that capacity is installed only on selected items, and for these there is an upper bound for expansion. Constraints (4) and (5) are flow conservation constraints. They also ensure that each commodity only flows during its respective time window. Constraints (6) ensure that the correct amounts of flow are sent from the sources, starting at the correct time. Constraints (7) and (8) are capacity constraints.

Aggregated problems can be of interest in practice because of their reduced size, which would allow them to be solved quicker than the original problem, and because they can provide us with interesting information about the instance or even solve the original problem. 


$$
\min \sum_{a \in A}\left(c_{a}^{1} y_{a}+c_{a}^{2} z_{a}\right)+\sum_{v \in N^{S}}\left(c_{v}^{1} y_{v}+c_{v}^{2} z_{v}\right)
$$

s.t.

$$
\begin{array}{rrr}
z_{a} \leq u_{a}^{+} y_{a} & r \\
z_{v} & \leq u_{v}^{+} y_{v} \\
\sum_{a \in \delta^{-}(v)} \phi_{a j}^{t}-\sum_{a \in \delta^{+}(v)} \phi_{a j}^{t}=0 & \forall v \in A \\
\sum_{a \in \delta^{-}(v)} \phi_{a j}^{t}-\sum_{a \in \delta^{+}(v)} \phi_{a j}^{t}=\phi_{v j}^{t}-\phi_{v j}^{t-1} & \forall j \in C, v \in N \backslash N^{S}, t \in\left[r_{j}, d_{j}\right] \cap \beta \\
\sum_{a \in \delta^{+}(q)} \phi_{a j}^{t}+\phi_{q j}^{t}=f_{j}^{q} & \forall j \in C, v \in N \backslash N_{j}^{S}, t \in\left[r_{j}, d_{j}\right] \cap \beta \\
\sum_{j \in C: t \in\left[r_{j}, d_{j}\right]} \phi_{a j}^{t} \leq(\bar{t}-t)\left(u_{a}+z_{a}\right) & \forall j \in C, q \in Q_{j}, t \in \beta: r_{j} \in[t, \bar{t}) \\
\sum_{j \in C: t \in\left[r_{j}, d_{j}\right]} \phi_{v j}^{t} \leq(\bar{t}-t)\left(u_{v}+z_{v}\right) & \forall a \in A, t \in \beta \\
y_{a}, y_{v} \in\{0,1\} & \\
z_{a}, z_{v} \geq 0 & \\
\phi_{a j}^{t} \geq 0 &
\end{array}
$$

Formulation 1. A natural time-expanded formulation for the Network Design Problem with $|T|$ time periods, accounting for time aggregations. Define $\beta \subseteq T$ as the ordered list of such breakpoints and let $\bar{t} \in \beta=$ $\{\ldots, t, \bar{t}, \ldots\}$ be the breakpoint found immediately after a breakpoint $t$ in $\beta$.

Theorem 1. The value of an optimal solution to an aggregated problem provides a lower bound on the value of an optimal solution to the fully disaggregated problem.

Proof. Consider a set of breakpoints $\beta=\{\ldots t, \bar{t}, \ldots\}$. From (6), if $r_{j}>t \in[t, \bar{t})$, then $j$ is allowed to be dispatched at $t$ rather than $r_{j}$. In the same way, if $d_{j}<(\bar{t}-1) \in[t, \bar{t})$, then $j$ is allowed to be delivered at $\bar{t}-1$ rather than $d_{j}$. Since in this case $(\bar{t}-t) \geq\left(r_{j}-d_{j}\right)$, it follows that $(\bar{t}-t) \sum_{a \in A}\left(u_{a}+z_{a}\right)+(\bar{t}-$ t) $\sum_{v \in N^{S}}\left(u_{v}+z_{v}\right) \geq\left(r_{j}-d_{j}\right) \sum_{a \in A}\left(u_{a}+z_{a}\right)+\left(r_{j}-d_{j}\right) \sum_{v \in N^{S}}\left(u_{v}+z_{v}\right)$. Therefore, since there could already be "extra" capacity being considered in the aggregated problem, we might not have to expand it as much as we would have to in the original problem. That would thus incur a smaller cost (objective function) for the obtained solutions.

\subsection{COMMODITY SHORTFALLS}

After solving an aggregated problem, we obtain tentative values for the design variables ( $y$ and $z$ ). These values are tentative because the installed capacity might not be sufficient to accommodate all the demand in the most congested time periods. To check if the tentative solution is feasible for the original problem, we can maximize the flow in the original network (with all time periods) for the tentative design, by fixing the design variables obtained with the aggregation and setting the objective function to maximize the sum of the flows going out of all source nodes in all time periods.

Equation 12 gives the portion of the demand of commodity $j \in C$ that could not be delivered given the installed capacity in the network, i.e., the shortfall

$$
\delta_{j}=F_{j}-\left(\sum_{a \in \delta^{-}\left(s_{j}\right)} \tilde{\phi}_{a j}^{d_{j}}+\tilde{\phi}_{s_{j} j}^{d_{j}-1}\right)
$$

Theorem 2. If $\delta_{j}=0 \forall j \in C$, then the capacity expansions are optimal for the fully disaggregated problem. 
Proof. Because, unlike the aggregated model, the max flow model considers all time indices, and enforce the original time windows for all commodities, the total available capacity in this problem is the same as the total available capacity in the original problem. From (1), it follows that each source dispatches at most $F_{j}$ for each commodity. If $\delta_{j}=0 \forall j \in C$, it means that exactly $F_{j}$ reached the target node at $d_{j}$ for all commodities and, therefore, the installed capacities were enough to transport all demand in time. In other words, there exists $\phi$ such that the fully disaggregated model is feasible for this particular set of design variables $y$ and $z$. Because of Theorem 1, in this case, the solution of the aggregation is feasible for the original problem, such a solution must be also optimal.

\section{A DISAGGREGATION SCHEME}

Algorithm 1 proposes a disaggregation scheme that, at each iteration, analyzes the commodity shortfalls of an aggregation and refines the aggregation by introducing a new breakpoint (or a set of new breakpoints).

Consider the time criticality $\chi_{t}$ of a time period $t \in T$ :

$$
\chi_{t}=\sum_{j: t \in\left[r_{j}, d_{j}\right]} \delta_{j}
$$

Note that whereas the commodity shortfall $\delta_{j}$ focuses on commodities, the time criticality $\chi_{t}$ focuses on time periods.

Because the value of $\chi$ can only change in time periods corresponding to a release date or a deadline of a commodity, breakpoints are only introduced at release dates and deadlines.

The algorithm stops either when the design variable values are feasible for the original problem $\left(\delta_{j}=0 \forall j \in\right.$ $C$ ), as the solution will be optimal, or when a sufficient amount of freight can already flow with the incumbent installed capacity (where sufficient can be defined based on the specific setting and goals).

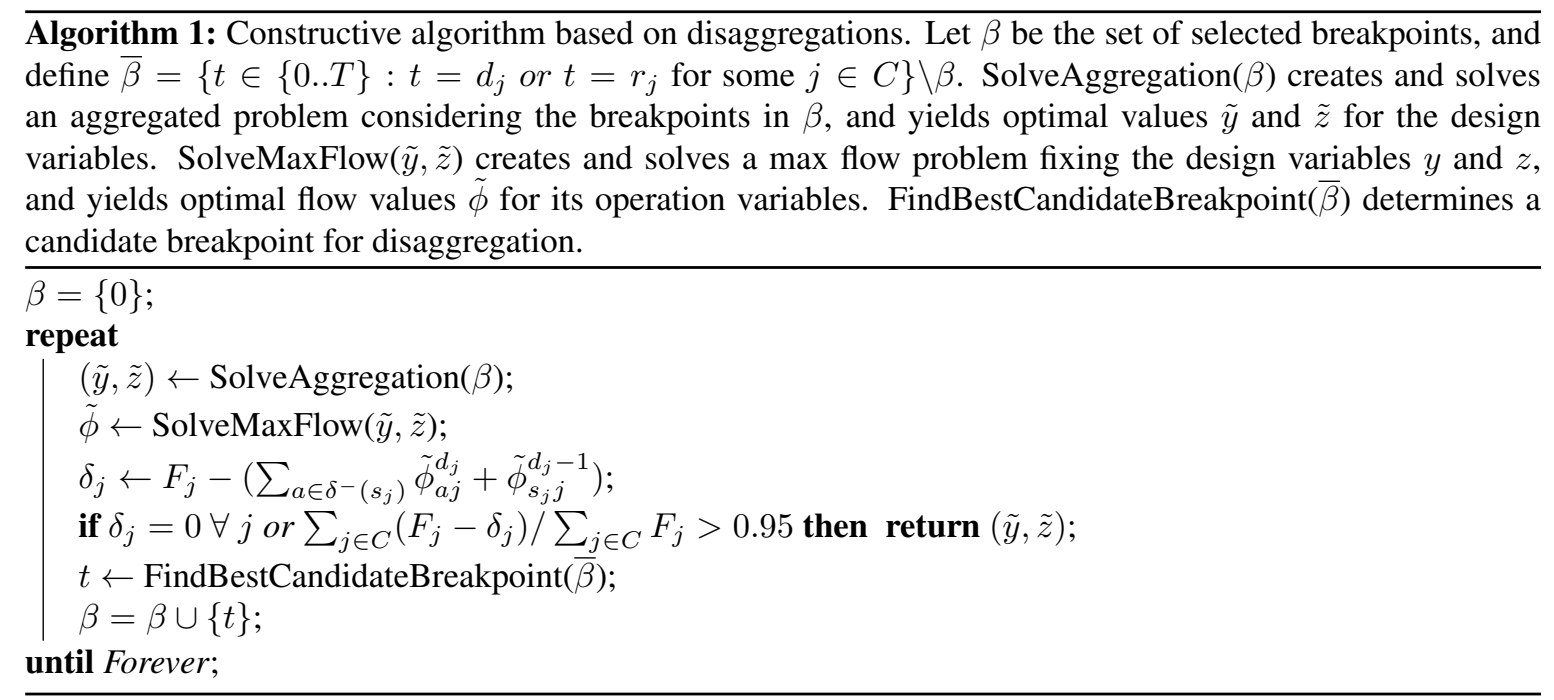

\subsection{FindBestCandidateBreakpoint $(\bar{\beta})$}

There are many possible strategies to select a time period to disaggregate at. In this research, we explore analyzing the incumbent solution's commodity shortfall and time criticality.

The first approach attempts to find a problematic commodity to be "isolated" at each iteration, and disaggregating at their release and due date, hoping to (indirectly) improve the maximum possible flow. One way to characterize such a commodity is by calculating $\operatorname{argmax}_{j \in C:\left\{r_{j}, d_{j}\right\} \cap \bar{\beta} \neq \emptyset}\left(\delta_{j}\right)$.

The second approach attempts to find a problematic time period directly and disaggregate around it. One way to characterize such a time period is by calculating $\operatorname{argmax}_{t \in \bar{\beta}}\left\{\chi_{t}\right\}$. 


\section{COMPUTATIONAL EXPERIENCE}

We use a test set with 18 instances divided in 6 sets with 3 different instances each, as detailed on Table 1 . The instances are derived from real scenarios observed in the HVCC. The underlying network structure contains 104 nodes and 129 arcs. Every commodity can be stored in four of the nodes (not necessarily the same nodes) and the difference between release date and deadline is no more than five days.

\begin{tabular}{|cccc|cccc|}
\hline Instance & Tonnage $(\mathrm{Mt})$ & $T$ & $|C|$ & Instance & Tonnage $(\mathrm{Mt})$ & $T$ & $|C|$ \\
\hline 1 & 90 & 120 & 336 & 10 & 90 & 360 & 990 \\
2 & 90 & 120 & 327 & 11 & 90 & 360 & 994 \\
3 & 90 & 120 & 331 & 12 & 90 & 360 & 997 \\
4 & 140 & 120 & 503 & 13 & 140 & 360 & 1491 \\
5 & 140 & 120 & 505 & 14 & 140 & 360 & 1474 \\
6 & 140 & 120 & 512 & 15 & 140 & 360 & 1472 \\
7 & 160 & 120 & 595 & 16 & 160 & 360 & 1666 \\
8 & 160 & 120 & 568 & 17 & 160 & 360 & 1650 \\
9 & 160 & 120 & 564 & 18 & 160 & 360 & 1662 \\
\hline
\end{tabular}

Table 1. Description of the test set. Each row represents a set. The columns represent the set number, the total tonnage, the number of days in planning period, and the number of commodities. Each set comprises three different instances.

The proposed methods were coded in python and ran on an Intel Core i7 laptop operating at 3.1GHz with 8GB of RAM. All MIPs and LPs were solved using CPLEX (v12.5, using only the default settings).

Table 2 summarizes the results obtained when simply trying to solve the instances with CPLEX without any aggregation with a time limit of one hour.

\begin{tabular}{|cc|cc|}
\hline Instance & Time $(\mathrm{s})$ & Instance & Time $(\mathrm{s})$ \\
\hline 1 & 8 & 10 & 181 \\
2 & 7 & 11 & 169 \\
3 & 7 & 12 & 139 \\
4 & 54 & 13 & Timeout \\
5 & 52 & 14 & 708 \\
6 & 60 & 15 & 846 \\
7 & 749 & 16 & Timeout \\
8 & 2092 & 17 & Timeout \\
9 & 1029 & 18 & Timeout \\
\hline
\end{tabular}

Table 2. Results obtained by simply attempting to solve the instances under consideration without any aggregation at all. Each row represents an instance set and every column (starting at the second) represents the running time for each instance in that set.

The results show, not surprisingly, that the problem becomes intractable when the number of time periods become large. Planning for one year using a daily granularity proves itself intractable using the natural MIP model.

To analyze the effectiveness of the proposed disaggregation schemes, we ran the algorithm 1 for each instance with the following settings: the algorithm stops when $95 \%$ of the total freight can be accommodated with the installed capacity, when one of the MIPs or LPs cannot be solved in an hour, or when the total time reaches four hours.

Table 3 details the number of time aggregations in the model when the installed capacity is sufficient to accommodate $95 \%$ of the freight for those instances that did not terminated because of a time limit. Recall that 
in each iteration two new breakpoints are introduced when using commodity defects and one new breakpoint is introduced when using time criticality to refine an aggregation.

\begin{tabular}{|ccc|ccc|}
\hline Inst & Size $(\delta)$ & Size $(\chi)$ & Inst & Size $(\delta)$ & Size $(\chi)$ \\
\hline 1 & 5 & 6 & 10 & 13 & 22 \\
2 & 9 & 16 & 11 & 17 & 43 \\
3 & 10 & 21 & 12 & 13 & 15 \\
4 & 3 & 8 & 13 & 11 & 30 \\
5 & 7 & 11 & 14 & 5 & 2 \\
6 & 9 & 14 & 15 & 7 & 16 \\
7 & 11 & 22 & 16 & - & - \\
8 & 9 & 11 & 17 & 5 & 7 \\
9 & 5 & 15 & 18 & - & 3 \\
\hline
\end{tabular}

Table 3. The number of breakpoints in the aggregation when $95 \%$ of the freight can be accommodated. A missing value indicates that the algorithm could not identify the desired network configuration within a reasonable amount of time.

The results are encouraging, but also show that more research and experimentation is needed. It is encouraging to see that for almost all instances it is possible in a few iterations, and thus with a very course aggregation, to identify a network configuration with installed capacity sufficient to accommodate $95 \%$ of the freight of the commodities.

However, these preliminary computational experiments also revealed that the convergence of the adaptive disaggregation procedure reduces dramatically in later iterations, when attempting to identify a network configuration with installed capacities sufficient to accommodate all the freight, thus leading to excessive run times. We could, however, obtain significant size reductions in every case. This is specially interesting when more time periods are considered. Since a significant part of the freight can be transported with the obtained solutions, and are therefore good quality bounds, these values can be used to hot-start a branch-and-cut procedure, improving its performance. Since these values are not expected to change significantly to be able to transport all the freight, further research would include the development of strategies to polish the obtained solutions to obtain the optimal, or at least good quality feasible solutions.

Another surprising observation, which is currently under further investigation, were the running times of the max flow solves, i.e., identifying the maximum amount of freight that can be accommodated with a certain network configuration. Even though the time-expanded network is large, we had expected faster run times. It may be necessary to switch from a general purpose LP solver for a purpose-built max flow solver.

\section{CONCLUSIONS AND FINAL REMARKS}

Dynamically adjusting time aggregations is a potentially powerful technique, especially for handling situations in which the natural optimization models get too large. Our preliminary computational experience with such approaches is encouraging, but also demonstrates that more research is necessary.

It is critically important to choose breakpoints wisely and perform as few iterations as possible. Therefore, further work would include the investigation of additional strategies to choose breakpoints to disaggregate at, of strategies that identify multiple breakpoints in each iteration, and use more information from the incumbent solutions.

\section{REFERENCES}

Ahmed, S., A. J. King, and G. Parija (2003, May). A Multi-Stage Stochastic Integer Programming Approach for Capacity Expansion under Uncertainty. Journal of Global Optimization 26(1), 3-24.

Floudas, C. A. and X. Lin (2004, October). Continuous-time versus discrete-time approaches for scheduling of chemical processes: a review. Computers \& Chemical Engineering 28(11), 2109-2129.

Luss, H. (1982, September). Operations Research and Capacity Expansion Problems: A Survey. Operations Research 30(5), 907-947. 
N. Boland et al., Time Aggregation for Network Design to Meet Time-Constrained Demand

Newman, A. M. and M. Kuchta (2007, January). Using aggregation to optimize long-term production planning at an underground mine. European Journal of Operational Research 176(2), 1205-1218.

Ordóñez, F. and J. Zhao (2007, September). Robust capacity expansion of network flows. Networks 50(2), 136-145. 